\title{
SUPPORTING INFORMATION Scaling Laws in the Diffusive Release of Neutral Cargo from Hollow Hydrogel Nanoparticles: Paclitaxel-Loaded Poly(4-vinylpyridine)
}

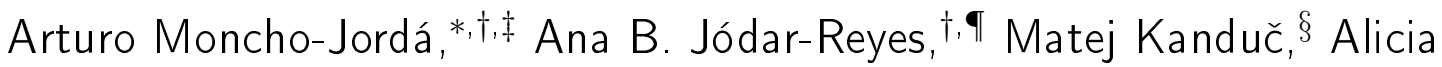
Germán-Bellod, ${ }^{\dagger}$ Juan M. López-Romero, $\|$ Rafael Contreras-Cáceres, ${ }^{\perp}$ Francisco Sarabia," Miguel García-Castro," Héctor A. Pérez-Ramírez,\# and Gerardo Odriozola*,\#

$\dagger$ Departamento de Física Aplicada, Universidad de Granada, Campus Fuentenueva S/N, $180{ }^{\prime} 1$ Granada, Spain.

$\ddagger$ Instituto Carlos I de Física Teórica y Computacional, Facultad de Ciencias, Universidad de Granada, Campus Fuentenueva S/N, 18071 Granada, Spain.

IExcellence Research Unit "Modeling Nature" (MNat), Universidad de Granada, Campus Fuentenueva S/N, 18071, Granada, Spain.

§Jožef Stefan Institute, Jamova 39, SI-1000 Ljubljana, Slovenia.

|| Departamento de Química Orgánica, Facultad de Ciencias, Universidad de Málaga, 29071 Málaga, Spain.

$\perp$ Departamento de Química en Ciencias Farmacéuticas, Facultad de Farmacia, Universidad Complutense de Madrid, Plaza Ramón y Cajal, 28040 Madrid, Spain. \# Física de Procesos Irreversibles, Ciencias Básicas e Ingeniería, Universidad Autónoma

Metropolitana-Azcapotzalco, Av. San Pablo 180, 02200 Ciudad de México, Mexico

E-mail: moncho@ugr.es; godriozo@azc.uam.mx

Part A: Calculation of the cargo time-dependent density profile using Dynamical Density Functional Theory (DDFT)

In this section we describe the dynamical density functional theory (DDFT) used to investigate the out-of-equilibrium diffusive release of Brownian interacting particles. ${ }^{1,2}$ Starting from an initial nonequilibrium state, the DDFT provides the time evolution of the cargo concentra- tion, $\rho_{\mathrm{c}}(r, t)$. The DDFT differential equation makes use of the space-dependent effective interaction of cargo molecules with the hydrogel and effective diffusion coefficient described in the main text $\left(u_{\text {eff }}(r)\right.$ and $D_{\text {eff }}(r)$, respectively).

For the particular case of spherical symmetry, it reads

$$
\frac{\partial \rho_{\mathrm{c}}(r, t)}{\partial t}=-\frac{1}{r^{2}} \frac{\partial}{\partial r}\left(r^{2} J_{\mathrm{c}}(r, t)\right)
$$

where $J_{\mathrm{c}}(r, t)$ is the time-dependent net flux at distance $r$ from the center of the hollow hydrogel. It can be written in terms of the gradient 
of the cargo chemical potential $\left(\mu_{\mathrm{c}}\right)$,

$$
J_{\mathrm{c}}(r, t)=-D_{\mathrm{eff}}(r) \rho_{\mathrm{c}}(r, t) \frac{\partial \beta \mu_{\mathrm{c}}(r, t)}{\partial r},
$$

where $D_{\text {eff }}(r)$ is the effective position-dependent cargo diffusion coefficient. Within the DDFT approach, $\mu_{\mathrm{c}}(r, t)$ is given by the functional derivative of the equilibrium free energy of the system, ${ }^{3,4} \mu_{\mathrm{c}}(\vec{r}, t)=\delta F\left[\rho_{\mathrm{c}}(\vec{r}, t)\right] / \delta \rho_{\mathrm{c}}(\vec{r}, t)$. We propose the following expression for $\mu_{\mathrm{c}}$, which has been successfully used in similar situations: ${ }^{5,6}$

$$
\begin{gathered}
\beta \mu_{\mathrm{c}}(r, t)=\ln \left(\rho_{\mathrm{c}}(r, t) \Lambda_{\mathrm{c}}^{3}\right)+\beta u_{\mathrm{eff}}(r) \\
+\phi_{\mathrm{c}}(r, t) \frac{8-9 \phi_{\mathrm{c}}(r, t)+3 \phi_{\mathrm{c}}(r, t)^{2}}{\left(1-\phi_{\mathrm{c}}(r, t)\right)^{3}},
\end{gathered}
$$

where $\Lambda_{\mathrm{c}}$ is the thermal wavelength of the cargo molecule. The first term corresponds to the ideal contribution. The second one is the effective interaction between the cargo molecule and the hydrogel nanoparticle. The third term accounts for the excluded-volume repulsion among cargo molecules. We assumed that they behave as hard spheres with radius $R_{\mathrm{c}}$, so the third term has been approximated by the Carnahan-Starling chemical potential, ${ }^{4}$ where $\phi_{\mathrm{c}}(r, t)=\frac{4}{3} \pi R_{\mathrm{c}}^{3} \rho_{\mathrm{c}}(r, t)$.

Note that this theoretical model improves other simpler theories based on the diffusion equation in two aspects. On the one hand, it includes cargo-cargo and cargo-hydrogel interactions. On the other hand, DDFT can also handle a position-dependent $D_{\text {eff }}(r)$, which experiences a significant reduction when the cargo diffuses into the polymer network. Note that cargo-cargo interactions turn important only when the local cargo concentration is high, such as the one appearing in the internal interface of attractive shells (Fig. 2(c) in the main text). In this case, accounting for these interactions is crucial to capture the possible blockage of the polymer shell. This should occur for a combination of strong polymer-cargo attraction and high initial cargo concentration.

The DDFT differential equation is solved with the following three boundary conditions. First, the flux in the center of the hydrogel must be zero because of the spherical symmetry, $J_{\mathrm{c}}(r=$ $0, t)=0$. Second, for a very diluted suspension of hydrogel particles, the cargo concentration far away from the hydrogel tends to zero, $J_{\mathrm{c}}(r \rightarrow \infty, t)=0$. We assume that a distance $r_{\infty}=5 b$ is already large enough to apply this boundary condition. Third, at $t=0$ all the cargo molecules are encapsulated inside the internal void of the hollow hydrogel, so $\rho_{\mathrm{c}}(r)=\rho_{\mathrm{c}}^{\mathrm{cav}}$ for $r \leq a-2 \delta$, and $\rho_{\mathrm{c}}(r)=0$ for $r>a-2 \delta$.

To solve the time-dependent DDFT differential equations, distances were scaled by $l_{0}=$ $1 \mathrm{~nm}$, and time by $\tau_{0}=l_{0}^{2} / D_{0}$, where $D_{0}=$ $k_{\mathrm{B}} T /\left(6 \pi \eta R_{\mathrm{c}}\right)$ is the cargo diffusion coefficient in bulk solution (of the order of $10^{-10} \mathrm{~m}^{2} / \mathrm{s}$ for the studied cargo sizes). In order to shorten the computation time of the numerical resolution, a nonuniform spatial grid was used to sample the distance $r$ : a smaller grid size is required at both hydrogel interfaces, where the gradients of $D_{\text {eff }}(r)$ and $u_{\text {eff }}(r)$ are larger, whereas thicker size intervals are employed in the regions inside and outside the hydrogel. In our calculations we chose $\Delta r_{\min }=0.02 l_{0}$. On the other hand, a time step of $\Delta t=10^{-4} \tau_{0}$ was used in all the calculations. This value is smaller than $\left(\Delta r_{\min }\right)^{2} /\left(2 D_{0}\right)$, thus preventing the appearance of unphysical sawtooth instabilities.

\section{Part B: Comments on the model's assumptions}

There are several assumptions involved in the theoretical model. In this section, we address the monomer and cargo sphericities, the hardcore and square-well potentials, and the continuum approximation for a uniform dielectric constant.

\section{Monomer and cargo sphericities}

A great advantage of assuming spherical cargo molecules and straight polymer chains is that one can find exact analytical expressions for the available volume inside the polymer network, which determines the steric exclusion potential (eq 8 of the main manuscript). However, the 
real available volume inside the polymer matrix is indeed affected by the cargo anisotropy, the internal structure of the polymer chains, the swelling state, or even the chain's thermal fluctuations. Despite its simplicity, eq 8 works fairly well in describing the excluded-volume effects in simulated polymer hydrogel, as shown elsewhere. $^{7}$

It should also be mentioned that it is not trivial to predict the effect of cargo anisotropy in the calculations. For instance, increasing the anisotropy of the cargo molecule should lead to a larger polymer-cargo attraction by increasing the contact area, but it should also decrease its accessible volume. In general, the overall effect cannot be predicted, but it depends on the specific cargo-polymer interaction per unit area. Thus, the assumption of spherical cargo molecules may lead to an overestimation or underestimation of the effective polymer-cargo potential.

\section{Hard-core and square-well poten- tial}

Our model assumes that the short-range interaction between the cargo molecule and the monomeric units of the polymer chains is given by a hard-core potential. For the case of attractive polymer layers, we add to the hard-core a square-well attraction (eq 9 in the main paper). Both potentials are idealizations that capture, in a simple way, the volume exclusion exerted by the polymer chains and the existence of a short-range attraction. With these simplifications, we are able to write analytical expressions for the effective interaction between the cargo molecule and the hydrogel nanoparticle, $u_{\text {eff }}(r)$. However, this simplification does not represent a risk or a decrease in the accuracy of our model. In fact, these approximated potentials work well for short-range interactions, given that the cargo radius, $R_{c}$, the attraction strength, $\epsilon$, and the attraction range, $\Delta$, are taken as effective parameters. These parameters can be chosen to reproduce the second virial coefficient of the real cargo-polymer interaction or any other relevant thermodynamic property.

\section{Uniform permittivity}

Although very common, the assumption of a uniform background with a fixed dielectric constant is another approximation. It is known that the dielectric constant inside a hydrogel can experience a significant decrease in the collapsed state. Indeed, the volume fraction of polymer inside a swollen hydrogel is very low, and the value of $\epsilon$ is close to that of the pure solvent (water), $\epsilon_{0}$, while in the collapsed state it is close to that of the dry polymer. This effect is expected to become important for charged cargo molecules. ${ }^{8}$ In this case, the electrostatic interaction leads to a Born electrostatic contribution to $u_{\text {eff }}(r)$.

For neutral cargo molecules, this approximation only modifies the ionic profiles inside and close to hydrogel nanoparicle. This, in turn, affects the osmotic term of the effective interaction (see eq 7 of the manuscript). Nonetheless, this contribution to $u_{\text {eff }}(r)$ is small for the conditions we study in the manuscript. Therefore, the consequence of considering the permittivity as a constant number is not relevant in the present work, as this approximation does not significantly modify $u_{\text {eff }}(r)$.

\section{Validity of the DDFT method}

Finally, we briefly discuss about the validity of the DDFT method used to predict the timedependent density profiles. This method relies on the so-called adiabatic approximation, which assumes that the nonequilibrium pair correlations of the real system are approximated by those of a fictitious equilibrium system, whose density distribution is given by the instantaneous density of the nonequilibrium system. This implies that the energy dissipation induced by the nonequilibrium fluxes neglects particle interactions. As a consequence of this limitation, DDFT is not able to capture the physics of systems that present memory effects in time, such as glass transitions, where DDFT underestimates the structural relaxation time scale. ${ }^{9,10}$ Since our particular diluted system of diffusing cargo is far from these conditions, it is expected to perform well, as it has been re- 
cently shown in previous studies of similar systems. ${ }^{5,6,11}$

\section{References}

1. Marconi, U. M. B.; Tarazona, P. Dynamic Density Functional Theory of Fluids. J. Chem. Phys. 1999, 110, 8032-8044.

2. Wu, J.; Li, Z. Density-Functional Theory for Complex Fluids. Annu. Rev. Phys. Chem. 2007, 58, 85-112.

3. Evans, R. In Fundamentals in Inhomogeneous Fluids; Henderson, D., Ed.; Marcel Derrer, Inc.: New York, NY 10016, USA, 1992; Chapter Density Functionals in the Theory of Nonuniform Fluids, pp 85-176.

4. Hansen, J.-P.; McDonald, I. Theory of Simple Liquids, 4th Edition; Academic Press: Oxford OX5 1GB, UK, 2013.

5. Angioletti-Uberti, S.; Ballauff, M.; Dzubiella, J. Dynamic Density Functional Theory of Protein Adsorption on PolymerCoated Nanoparticles. Soft Matter 2014, 10, 7932-7945.

6. Moncho-Jordá, A.; Germán-Bellod, A.; Angioletti-Uberti, S.; Adroher-Benítez, I.; Dzubiella, J. Nonequilibrium Uptake Kinetics of Molecular Cargo into Hollow Hydrogels Tuned by Electrosteric Interactions. ACS Nano 2019, 13, 1603-1616.

7. Ahualli, S.; Martín-Molina, A.; QuesadaPérez, M. Excluded Volume Effects on Ionic Partitioning in Gels and Microgels: A Simulation Study. Phys. Chem. Chem. Phys. 2014, 16, 25483-25491.

8. A.R. Khokhlov, E. K., K.B. Zeldovich In Electrostatic Effects in Soft Matter and Biophysics; C. Holm, R. P., P. Kékicheff, Ed.; Springer Science+Business Media: Dordrecht, The Netherlands, 2001; Chapter Counterions in Polyelectrolytes, pp 283315 .
9. Schmidt, M.; Brader, J. M. Power Functional Theory for Brownian Dynamics. J. Chem. Phys. 2013, 138, 214101.

10. Reinhardt, J.; Weysser, F.; Brader, J. M. Density Functional Approach to Nonlinear Rheology. EPL (Europhysics Letters) 2013, 102, 28011.

11. Angioletti-Uberti, S.; Ballauff, M.; Dzubiella, J. Competitive Adsorption of Multiple Proteins to Nanoparticles: The Vroman Effect Revisited. Mol. Phys. 2018, 116, 3154-3163. 